\title{
The 2014 ABJS Nicolas Andry Award: The Puzzle of the Thumb: Mobility, Stability, and Demands in Opposition
}

\author{
Amy L. Ladd MD, Joseph J. Crisco PhD, \\ Elisabet Hagert MD, PhD, Jessica Rose PhD, \\ Arnold-Peter C. Weiss MD
}

Received: 30 September 2013/Accepted: 14 August 2014/Published online: 30 August 2014

(C) The Association of Bone and Joint Surgeons ® 2014

\begin{abstract}
Background The paradoxical demands of stability and mobility reflect the purpose and function of the human thumb. Its functional importance is underscored when a thumb is congenitally absent, injured, or afflicted with degenerative arthritis. Prevailing literature and teaching implicate the unique shape of the thumb carpometacarpal (CMC) joint, as well as its ligament support, applied forces, and repetitive motion, as culprits causing osteoarthritis
\end{abstract}

One or more of the authors has received, during the study period, funding from the Williams Charitable trust (ALL), Orthopaedic Research and Education Foundation educational grant (ALL, JR), NIH (SBIR)/NBIB R43 EB003067-01Al, and 02A1 (ALL), NIH/ SBSR 1R01AR059185-01A1 (ALL, JJC, APCW), RJOS/OREF/ DePuy Career Development Award (ALL), Packard Foundation grant (ALL, JR), and 2011 American society for Surgery of the Hand resident seed grant (ALL).

All ICMJE Conflict of Interest Forms for authors and Clinical Orthopaedics and Related Research ${ }^{\mathbb{R}}$ editors and board members are on file with the publication and can be viewed on request.

The primary work was performed at Stanford University Palo Alto, CA, USA) and recent kinematic CT work as cited was performed at Brown University (Providence, RI, USA) and Stanford University (Palo Alto, CA, USA).

Electronic supplementary material The online version of this article (doi:10.1007/s11999-014-3901-6) contains supplementary material, which is available to authorized users.

\section{A. L. Ladd ( $\bowtie)$}

Department of Orthopaedic Surgery, Stanford University, Chase Hand Center, 770 Welch Road, Suite 400, Palo Alto, CA 94304, USA

e-mail: alad@stanford.edu

J. J. Crisco, A.-P. C. Weiss

Department of Orthopaedics, Warren Alpert Medical School of Brown University and Rhode Island Hospital, Providence, RI, USA
(OA). Sex, ethnicity, and occupation may predispose individuals to OA.

Questions/purposes What evidence links ligament structure, forces, and motion to progressive CMC disease? Specifically: (1) Do unique attributes of the bony and ligamentous anatomy contribute to OA? (2) Can discrete joint load patterns be established that contribute to OA? And (3) can thumb motion that characterizes $\mathrm{OA}$ be measured at the fine and gross level?

Methods We addressed the morphology, load, and movement of the human thumb, emphasizing the CMC joint in normal and arthritic states. We present comparative anatomy, gross dissections, microscopic analysis, multimodal imaging, and live-subject kinematic studies to support or challenge the current understanding of the thumb CMC joint and its predisposition to disease.

Results The current evidence suggests structural differences and loading characteristics predispose the thumb CMC to joint degeneration, especially related to volar or central wear. The patterns of degeneration, however, are not consistently identified, suggesting influences beyond inherent anatomy, repetitive load, and abnormal motion.

Conclusions Additional studies to define patterns of normal use and wear will provide data to better characterize $\mathrm{CMC} \mathrm{OA}$ and opportunities for tailored treatment,

\footnotetext{
E. Hagert

Hand \& Foot Surgery Center, Karolinska Institutet, Stockholm, Sweden

J. Rose

Department of Orthopaedic Surgery, Motion \& Gait Analysis Laboratory, Lucile Packard Children's Hospital, Palo Alto, CA, USA
} 
including prevention, delay of progression, and joint arthroplasty.

\section{Introduction}

The opposable thumb, unique to our species, is one of our essential defining traits. In concert with the human brain, it orchestrates masterful tasks with refined flexibility, creating mobility and stability in paradox. The thumb works in precise coordination with the fingers, whether poised to grasp a fistful of arrows or pen the perfect opening line. The absence, injury, and loss of this critical digit are, appropriately, important topics to surgeons.

Classical treatises and qualitative investigations have been devoted to its purpose and function $[9,21,34,35$, 105]. The current literature suggests a myriad of etiologies as to why thumb carpometacarpal (CMC) osteoarthritis $(\mathrm{OA})$ is so common, including prevalent theories of anterior oblique ligament degeneration, ligamentous laxity, hormonal changes with menopause, genetic predisposition, repetitive use, and abnormal load transmission [4, 34-36, $53,85-89,107,108]$. But to date, the absence of quantitative biomechanical, live-subject, and large population studies has impaired formation of a consensus on this topic.

Our research program has approached the thumb CMC by investigating the morphology, forces, and motion of both normal and arthritic joints in an effort to provide quantitative evidence of disease progression. We present comparative anatomy, gross dissections, microscopic analysis, multimodal imaging, and live-subject kinematic studies. Our recent studies address the following questions: (1) Do unique morphological attributes of the bony and ligamentous anatomy contribute to OA? (2) Can discrete joint load patterns be established that contribute to OA? And, finally, (3) can thumb motion that characterizes OA be measured at the fine and gross level? We have provided an overview with references of the image modalities presented in this manuscript (Table 1).

\section{Morphology}

\section{Functional Morphology}

From an evolutionary standpoint, the thumb (from the Dutch word "duim," meaning stout; the related Latinderived terms used in anatomic nomenclature come from the Latin word "pollex," meaning powerful) has progressively receded in length, yet gained in girth and might. To oppose its neighbors with stable pinch and grasp, it recessed more than millions of years from a three-phalanx digit to the current two phalanges and metacarpal on a mobile saddle joint $[21,30,37-39,44,46,58,69,71]$.
With the trapezium's terminal post at the radial carpal arch establishing the thumb's offset position, its coordinates lie tangential to the fingers (Fig. 1) [26, 108]. The concavoconvex saddle trapezial surface creates "reciprocal reception" [44] with the metacarpal to permit wide circumduction. The larger ulnar surface and eccentric contact provides a "rollback" or "screw-home" mechanism, similar to a knee $[15,16,31,36]$. Composite opposition to hold a pen requires positioning and stabilizing muscles across the CMC joint, imparting abduction, flexion, translation, and pronation; additional contributors are the metacarpophalangeal (MP) and interphalangeal joints [16].

Primate comparative anatomy and the scant evidence from early hominins suggest the human saddle joint affords more motion with its shallower, looser design and robust complement of intrinsic muscles [1, 2, 52, 56, 69, 102]. CMC arthritis in great apes and other primates is unknown, although other wrist arthritis is common in knuckle-bearing gorillas [93]. We recently performed CT segmentation of an elderly male gorilla, visualizing advanced arthritis at the trapezial-trapezoidal and second CMC joint. The elderly (age 44 years) gorilla's CMC joint is more constrained than human and not part of either weightbearing ambulation or precise manipulative activity (Fig. 2A-B) (PDF 1; Supplemental material is available with the online version of $C O R R^{\circledR}$ ). In the gorilla at least, this suggests load transmission and function influence degeneration. Human prehension requires an opposable strong thumb, the small finger's hypothenar complex [58], and wrist positioning in the "dart-thrower's motion" [27, 84] with thumb and forearm in line, extending the lever arm and imparting mechanical advantage to throw a fastball or swing a golf club $[69,105]$.

With congenital anomalies, a stable CMC joint is the defining characteristic of a functioning thumb; in fact, an unstable and incomplete basal joint in the hypoplastic thumb is the primary reason to remove the thumb and proceed with pollicization $[14,20,68]$. The pollicized index finger performs well with a rotated metacarpal head as its new trapezium. We do not have the demographic information to understand whether the converted CMC (the former MP joint) degenerates with the mobile and stable demands of pinch and grasp like the susceptible saddle joint, but we can argue that a simplified joint performs reasonably well (Fig. 3A-G) (Video 1; Supplemental material is available with the online version of CORR $^{\mathbb{R}}$ ).

\section{Osteology}

The cartilaginous roundness of carpal bone growth and development progresses to the prominences and recesses of adulthood, possessing variable surface characteristics within populations and sexes [3, 4, 70, 85, 88, 107]. 


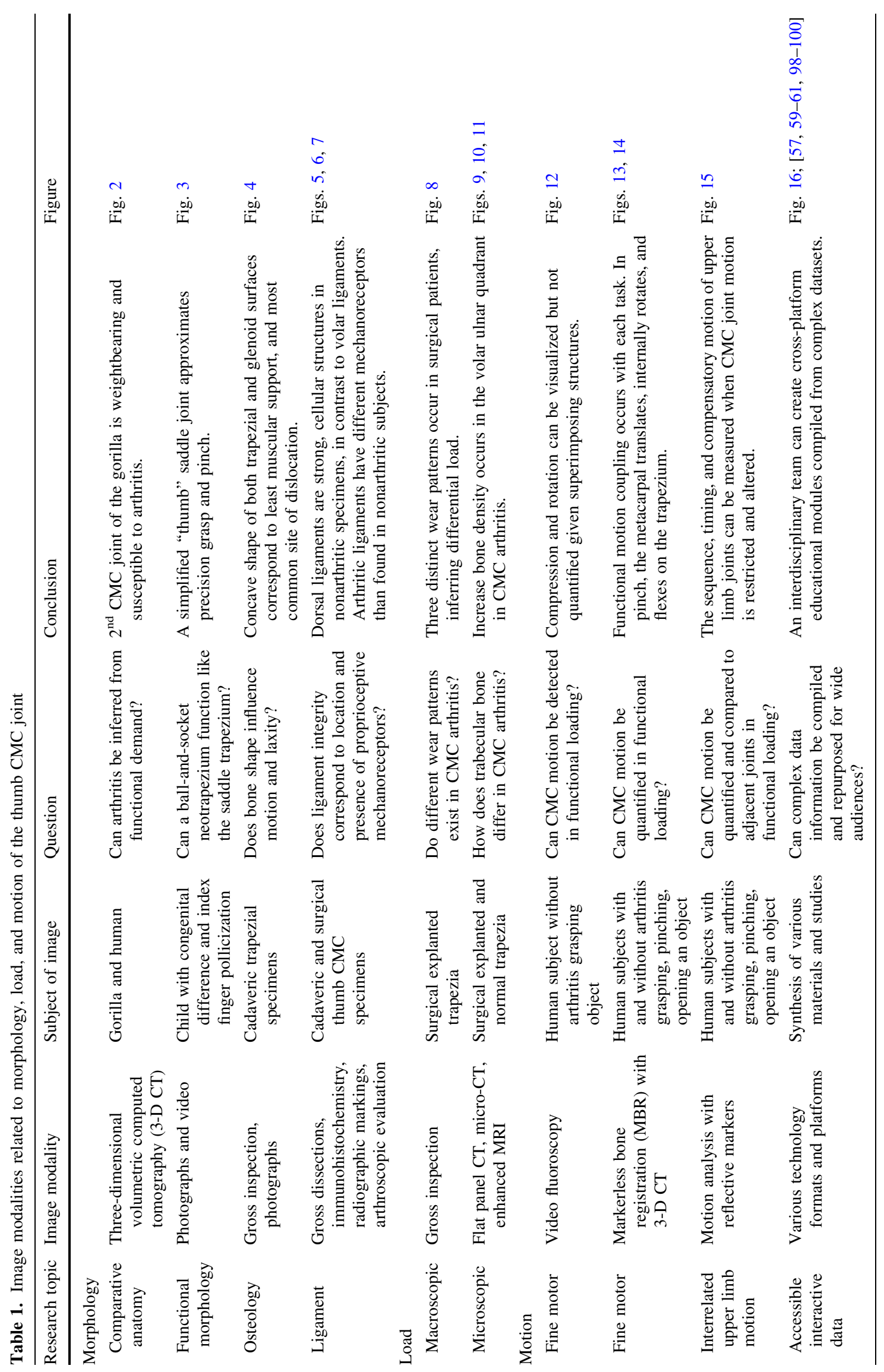




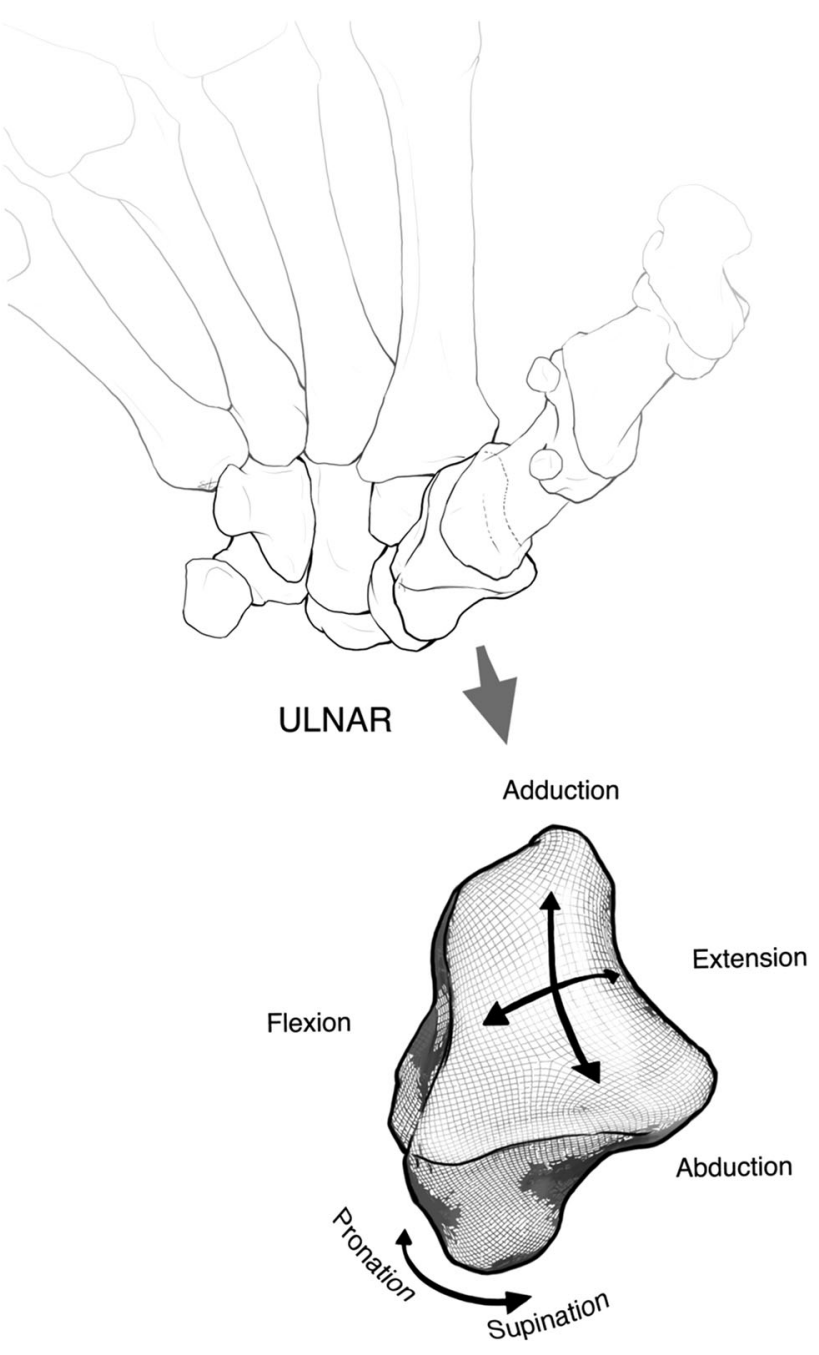

RADIAL

Fig. 1 The motion arcs of the metacarpal on the trapezium are flexion-extension and abduction-adduction. Pronation-supination represents composite rotation and translation of this triaxial joint based on morphology and muscular activity $[26,30,37,38,46]$. The thumb position in relation to the fingers represents a completion of the carpal arch, which places the CMC joint obliquely volar to the adjacent fingers and proximally oriented on its radial aspect. The arcs of motion thus are out of phase with the fingers. Image redrawn from CT surface rendering of a normal right hand. Published with kind permission of (c) S. Hegmann 2014. All Rights Reserved.

Although increased trapezial tilt is prevalent in radiographic arthritis [13], this may reflect trapezial-trapezoid articulation laxity [42]. In our recent evaluation of 82 trapeziae [64, 65, 103], we confirm that the trapezium's metacarpal surface is gently comma-shaped (left) or $\mathrm{C}$-shaped (right) with the ulnar-radial axis as vertical reference (Fig. 4A-B). This compares to the eccentrically shaped glenoid, also comma-shaped (left) or C-shaped (right); both joints have a loose configuration relying on ligament and muscular stability, tangential to the plane of the thorax and fingers, respectively. In both the trapezium and glenoid, the gentle concave curvature of the eccentric shape corresponds to the side of least muscular support, and common site of dislocation-anterior in the shoulder, and dorsal in the hand.

\section{Ligaments}

The CMC joint's investing ligaments are stout dorsally (Fig. 5A-F) and thin volarly (Fig. 6A-D), both as measured in thickness and cellular content $[45,62,65,75,109]$. We have verified their presence from outside-in with gross dissection, and from inside-out with arthroscopic evaluation (Fig. 7); additionally, we evaluated their radiographic position on 47 cadavers.

The dorsal deltoid complex emanates from the dorsal tubercle and distally fans across the dorsum of the metacarpal. The stout collagen is organized and cellular and primarily has the proprioceptive mechanoreceptors known as Ruffini endings present close to ligamentous attachments, demonstrated consistently in 10 normal specimens $[45,62,65]$. This supports earlier reports of the principal role of dorsal stabilizing ligaments [13, 36, 49, 50]. Conversely, the volar ligaments are thin, capsular structures, variable in location, with thenar muscles intimal to their presence. Whereas many investigators emphasize importance of the anterior oblique "beak" ligament in the presence and creation of arthritis [32-35, 85-89], our studies have focused on normal anatomy analysis, which may in part address this discrepancy. The volar ligaments were lacking in normal ligamentous structure and also mechanoreceptor innervation. Since the volar ligaments in our studies were deemed insufficient to provide static stability, we believe the thenar muscles, intimal to the volar capsule, are of primary importance in volar CMC joint stability. A followup study we performed examining the ligaments of 11 arthritic subjects at time of surgery (10 women, one man) confirmed the predominance of mechanoreceptors in the dorsal radial ligament and paucity in the anterior oblique ligament [75]. These subjects underwent surgery for symptomatic arthritis and thus may have altered neural and ligament structure compared to asymptomatic individuals; the causal relationship of symptoms, ligament pathology, and mechanoreceptors remains unclear.

Furthermore, the role of mechanoreceptors in supporting ligaments versus supporting muscles in the thumb CMC joint has not been determined. Muscle is rich in proprioceptive muscle spindles, and their presence in thenar muscles has been demonstrated with conditioning experiments in both central and peripheral 


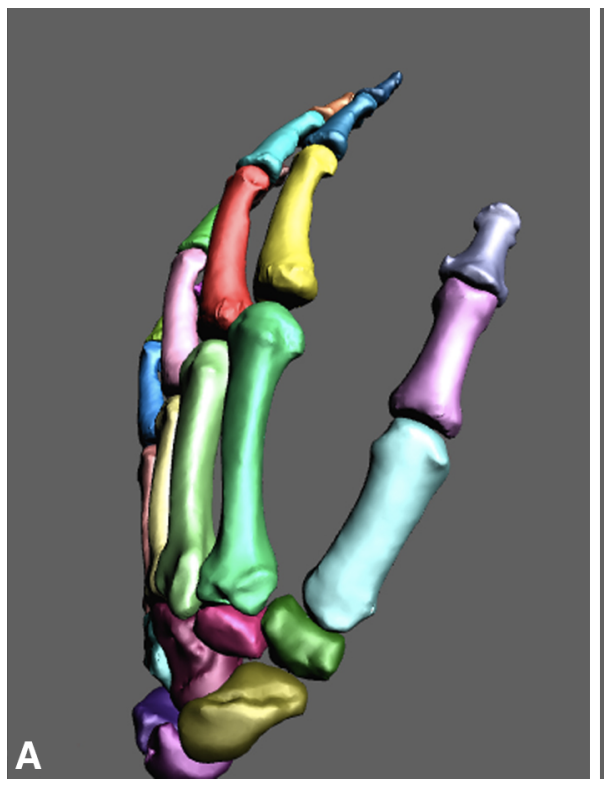

Fig. 2A-B A comparison of (A) a normal human hand to (B) a silver-backed gorilla (Gorilla gorilla) hand from CT surface renderings is shown. The elderly (age 44 years) gorilla's CMC joint is comparatively more constrained with a deeper articulating trapezial

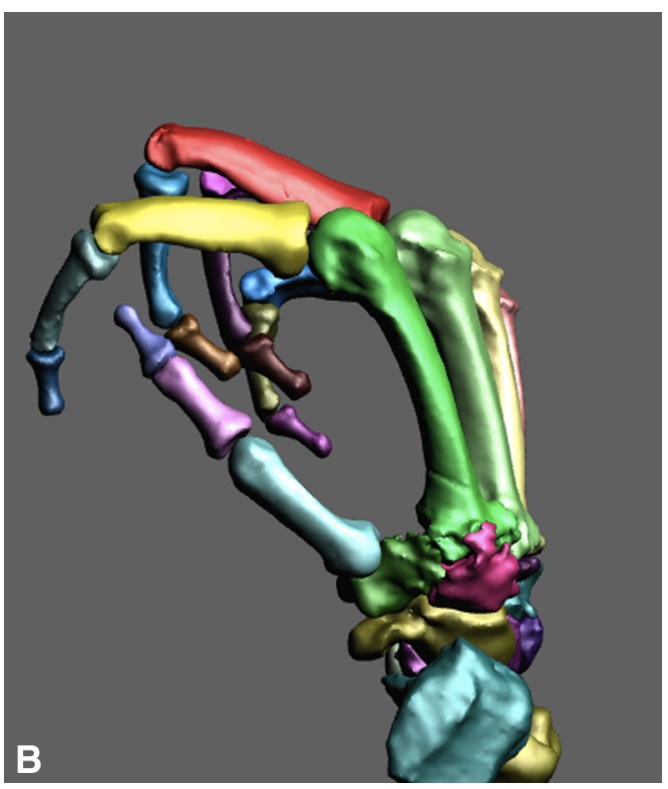

surface and more evenly matched in size compared to the human hand. Significant presence of trapezial-trapezoidal and second CMC OA is noted. Published with kind permission of (C) Ian Fitzgerald and Stanford University 2014. All Rights Reserved. evoked potential testing [95, 97]. These findings support thenar and complementary first dorsal interosseous strengthening in functional $\mathrm{CMC}$ rehabilitation with favorable MP flexed positioning, preventing hyperextension [78, 81, 90].

The ulnar complex creates a checkrein effect. The ulnar collateral ligament, or more appropriately, the volar trapeziometacarpal ligament, and the dorsal trapeziometacarpal ligament span from their more central locations proximally to a conjoined attachment directly ulnarly $[12,16]$. The stout ulnar complex may be critical to volar concentration of forces.

\section{Next Steps}

The height, width, and articular facet interdependence of the CMC joint and other trapezial articulations require further investigation, as well as examining different populations and stages of disease [42, 70]. The role of proprioception, first described by hand philosopher Sir Charles Bell as "muscle sense" with a feedback loop for voluntary control $[7,8]$, constitutes a future investigation-potentially analyzing the muscle characteristics, sarcomeres, and possibly proprioceptive endings in the thenar muscles of asymptomatic and symptomatic subjects.

\section{Load}

\section{Macroscopic}

Articular and trabecular wear patterns provide an inference of biomechanical loading [106]. The literature varies on reported load patterns in trapezial arthritis $[3,4,32,53,54$, $86,89]$, with volar wear predominating [86, 87, 89, 107].

Using reported location and wear classifications $[26,83$, 89, 107], we prospectively examined 36 surgically explanted trapeziae with advanced arthritis in 27 women $(75 \%)$ and nine men $(25 \%)$, with a mean age of 64 years (range, 33-76 years). We found three consistent patterns of wear: (1) A retained saddle in 17 specimens (47\%), (2) a "dish" concave pattern of wear in 12 specimens (33\%), and (3) a "cirque" pattern of an extra facet volarly in seven specimens (19\%) (Fig. 8), confirmed with micro-CT evaluation (Fig. 9A-C) [103]. Consistency was shown with a strong intrarater reliability (0.97) and interrater reliability (0.95). The retained saddle rarely had accompanying osteophytes or loose bodies, with cartilage remaining typically on the ulnar $1 / 2$. Volar osteophyte formation at the metacarpal beak articulation was present in all cirque specimens. The expansile dish pattern, in contrast, had significant rimming osteophytes and loose bodies. Surgical inspection did not reveal metacarpal dysmorphology with the dish pattern, other than extrinsic osteophytes and joint 

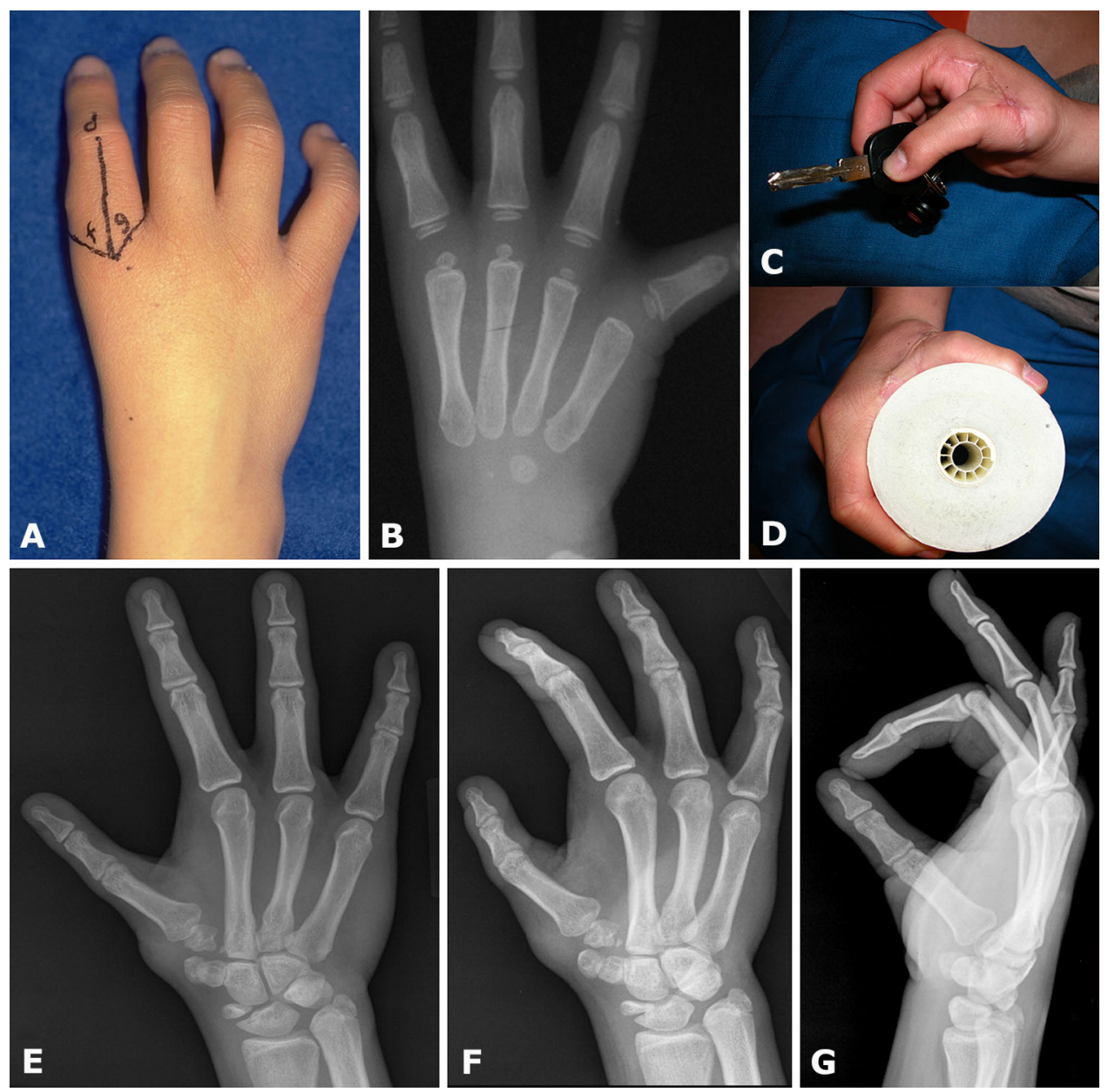

Fig. 3A-G A pollicized right index finger functions like a thumb. The (A) clinical and (B) radiographic appearance before pollicization is shown. The $(\mathbf{C}, \mathbf{D})$ clinical and $(\mathbf{E}-\mathbf{G})$ radiographic appearance 9 years after pollicization is also shown. The previous second

eburnation in the cases of dysplastic dish shape, similar to other reports $[79,80,86,107]$. This is unlike posttraumatic arthritis of a Rolando fracture, where the metacarpal is fractured and becomes dysplastic yet typically the trapezial shape is unaltered. This implies that posttraumatic and idiopathic degenerative OA create very different load demands on the CMC joint.

\section{Microscopic}

Areas of compressive loading have densely packed trabecular bone in volume and width. The vertebral bodies, hip, and knee are among the most widely studied with micro-CT, demonstrating dense subchondral bone and concentrated trabeculae $[6,17,18,29]$. The trapezium's middle $1 / 3$ studied with micro-CT in normal and arthritic specimens demonstrated higher metacarpal head, now the trapezium, has taken on duties of a saddle joint, with (E) change in morphology capable of (F) grasp and (G) pinch.

trabecular presence in the ulnar column of subchondral bone, with increased volume in the arthritic radial column [80].

We performed a complementary micro-CT study [48] evaluating quadrant $[26,108]$ trabecular characteristics in the trabecular midregion bone of 13 normal and 16 arthritic trapeziae from patients with mean ages of 69 and 61 years, respectively and male-to-female ratios of $4: 9$ and 10:6, respectively [64]. ANOVA with post hoc Bonferroni/Dunn correction revealed no difference in bone volume fraction between the OA and normal specimens $(p=0.31)$ although OA trapeziae demonstrated higher trabecular number $(p=0.025)$ and connectivity $(p=0.018)$ than non-OA trapeziae. The volar ulnar quadrant of both populations consistently revealed higher bone volume fraction, trabecular number, and connectivity than the dorsoradial and volar radial quadrants. This demonstrates additional evidence of preferential loading to support the thumb's 


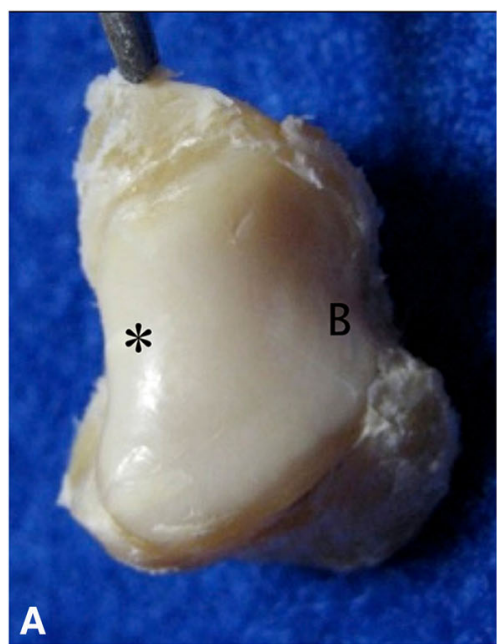

B

Fig. 4A-B (A) The left trapezium metacarpal surface, viewed from above, demonstrates a slight concave curve dorsally (marked $*$ ) and concave volarly, where the beak of the metacarpal articulates (marked B). The ulnar $1 / 2$ is eccentrically larger than the volar $1 / 2$. This appears as a comma shape when viewed from this perspective; conversely, a right trapezium has a gentle $\mathrm{C}$ shape. (B) This has the same general shape of the glenoid: The left is a comma-shaped and the right $\mathrm{C}$ shaped. Similar to the glenohumeral joint, the muscular bulk resides

anatomic juxtaposition to the fingers for grasp and pinch and suggests a stress concentration above the trapezoidal prominence.

\section{Next Steps}

We recently performed a systematic review of the utility of the Eaton radiographic classification to assess the reliability in grading disease severity [11]. According to the results of the review, interobserver reliability is poor to fair (kappa values: $0.11-0.56)$ and intraobserver reliability fair to moderate (kappa values: 0.54-0.657). This analysis provides the basis for creating a more reliable and predictive measurement. We have recently reported a Thumb Osteoarthritis Index obtained from a modified Robert's view [91], which may have utility in prospective analysis, demonstrating a high intraobserver and interobserver reliability using intraclass correlation coefficient evaluation (0.95 and 0.85 , respectively) among four observers of varied experience [unpublished data; presented at Association of Bone and Joint Surgeons Annual meeting 2013]. Our next steps include (1) performing prospective radiographic prediction of trapezial wear patterns before surgical trapeziectomy and (2) comparing articular cartilage thickness and subchondral bone of both trapezial and metacarpal articular surface in normal and arthritic live subjects. This will provide an indirect measurement of joint contact loading (Fig. 10A-D), using a flat-panel portable on the convex side, and more robust ligamentous support resides on the concave side. Common traumatic dislocations of the $\mathrm{CMC}$ joint and the glenohumeral joint occur on the sides with less musculotendinous support: Dorsal and anterior, respectively. This bony morphology and its soft tissue envelope suggest a contributing role to inherent stability. Figure $4 \mathrm{~B}$ modified from Gray $\mathrm{H}$, Lewis $\mathrm{WH}$ (ed). Anatomy of the Human Body. 20th ed. Philadelphia, PA: Lea \& Febiger; 1918.

CT scanner to correlate micro-CT and histology. Comparative standard CT with a clinical MRI (1.5 T) permits excellent complementary imaging, with further enhancement software of false-color assignment [10] that highlights characteristics suggestive of biochemical aberrations (Fig. 11A-B). Examining a live subject on a T3 scanner with a similar protocol, however, proved challenging since noise-to-signal ratio worsens as the acquisition field narrows. Exact registration of anatomic sites between multimodal imaging techniques (eg, CT, MRI, histology) with disparate datasets and algorithmic interpretation presents a computational challenge but remains a focus of our collective research efforts.

\section{Motion}

\section{Fine Motor}

At the discrete level of the CMC joint, the micromotion has been theorized $[16,36,77,108]$ but not quantified in humans, especially as a 3-D concept. Video capture with cine fluoroscopy provides high-resolution, albeit highradiation-linked, radiographs, whereas mini-image intensifiers provide fast imaging capabilities with resolution sacrifice (Fig. 12A-B) (Videos 2 and 3; Supplemental materials are available with the online version of $C O R R^{\circledR}$ ). Similar to plain radiographs, we rely on multiple views to provide a qualitative (3-D) construct. 

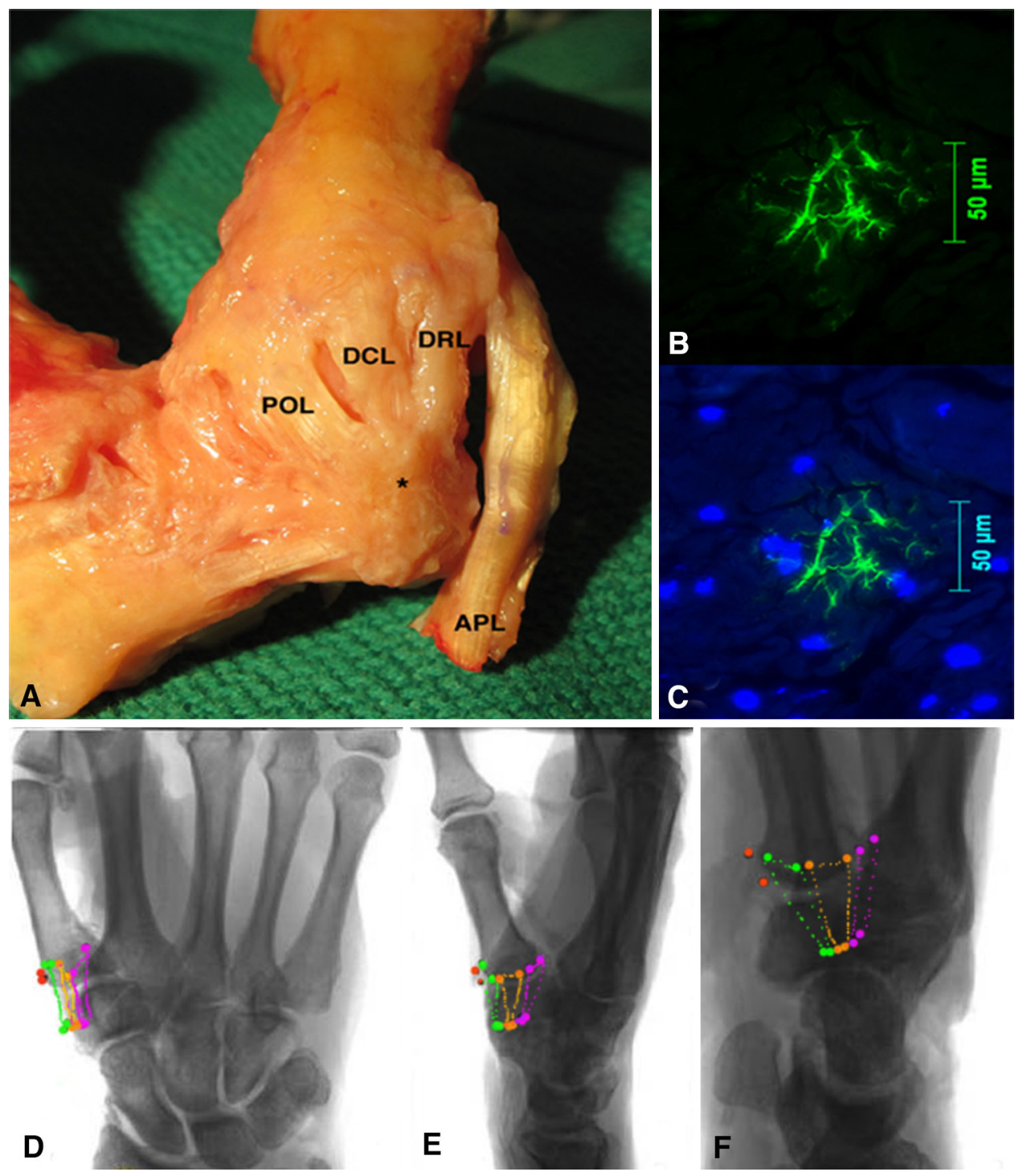

Fig. 5A-F The dorsal ligamentous anatomy from (A) gross dissections, $(\mathbf{B}, \mathbf{C})$ immunohistochemical staining, and (D-F) radiographic marking is shown. (A) In a left hand, the stout dorsal ligaments form a deltoid complex emanating from the dorsal tubercle $(*)$, representing the dorsal radial ligament (DRL), dorsal central ligament (DCL), and posterior oblique ligament $(\mathrm{POL}) . \mathrm{APL}=$ abductor pollicis longus. Dorsal ligament immunofluorescent protein gene product 9.5 and

Our coinstitutional collaboration currently uses a markerless bone registration technique for 3 -D in vivo kinematic analysis in an effort to quantify CMC micromotion and its correlation with $\mathrm{OA}$ presence and progression. We are applying the CT carpal kinematic protocol developed for evaluating carpal kinematics, including dart-thrower's motion [27, 47, 63], to study asymptomatic and subjects with early OA with the tasks of grasp, pinch, and jar opening using positioning jigs embedded with load cells. Our preliminary findings in 46 asymptomatic subjects ( 22 men, 24 women, each stratified 4',6-diamidino-2-phenylindole staining demonstrate (B) a Ruffini ending and (C) nucleated collagen. These were essentially absent in all volar ligaments examined. The radiographic representation of the dorsal ligaments with (D) AP, (E) lateral, and (F) Robert's views is shown: $\mathrm{DRL}=$ green $; \mathrm{DCL}=$ orange; $\mathrm{POL}=$ magenta $; \mathrm{APL}=$ red. Published with kind permission of (C) AL Ladd and E Hagert, 2014. All Rights Reserved.

into two age groups: $18-25$ years and $40-75$ years) in pinch, progressing from unloaded to loaded, demonstrated metacarpal volar translation, internal rotation, and flexion on the distal trapezial surface [47, 63]. This same progression in object grasp revealed the metacarpal undergoes ulnar translation, flexion, and abduction relative to the distal trapezial surface (Fig. 13). Functional coupling of CMC motion from the neutral position occurs with each task $(\mathrm{p}<0.001)$. The subjects did not vary between sexes in either age group, but the movement patterns differed statistically between them $(\mathrm{p}<0.001)$ [47]. 


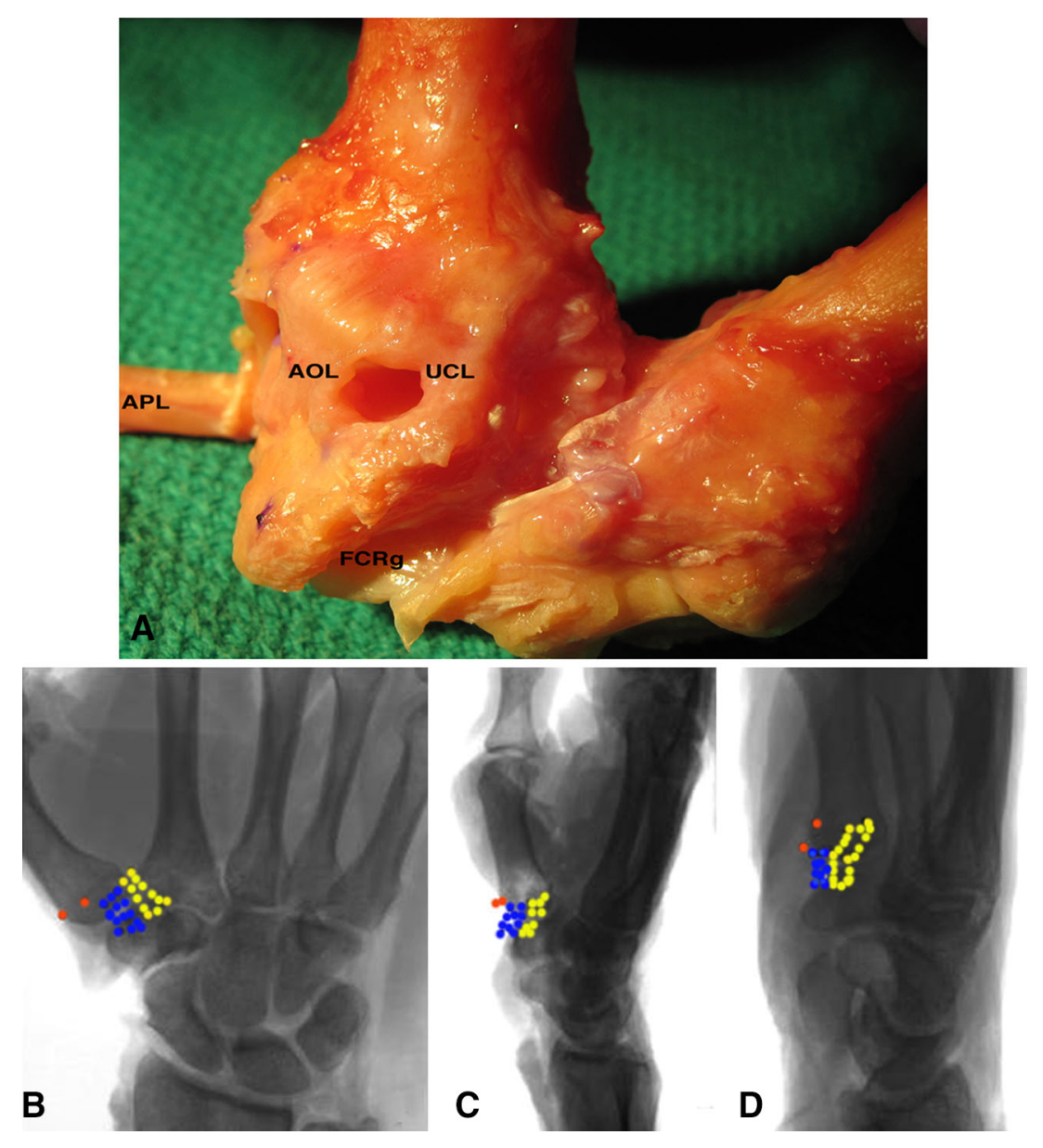

Fig. 6A-D The volar ligamentous anatomy from (A) gross dissections and (B-D) radiographic marking is shown. (A) The volar ligaments, anterior oblique ligament (AOL) and ulnar collateral ligament (UCL), in passive extension are shown. The window between the thin ligaments is commonly found, intimal to the thenar muscles. $\mathrm{APL}=$ abductor pollicis longus; $\mathrm{FCRg}=$ the obliquely

This consistent patterning provides a solid framework to further analyze the kinematics of subjects with early OA and whether changes in motion over time, including abnormal motion or increased laxity, may predict OA progression in symptomatic subjects with little evidence of radiographic disease (Fig. 14). These preliminary studies support our trabecular findings of preferential loading on the volar ulnar quadrant of the distal trapezial surface, where greatest contact occurs with each of these activities. Furthermore, the study of both asymptomatic and arthritic subjects underway (NIH/SBSR 1R01AR059185-01A1 coinstitutional grant, Brown University and Stanford University) will permit quantifying the shape, contour, facet size, and interrelating features that will address the "mismatch" and purported size differences reported in ex vivo methods [4, 53, 107]. oriented flexor carpi radialis groove. (D) The radiographic representation of the volar ligaments with (B) AP, (C) lateral, and (D) Robert's views is shown: $\mathrm{AOL}=$ blue; $\mathrm{UCL}=$ yellow; $\mathrm{APL}=$ red. Published with kind permission of (C) AL Ladd and E Hagert, 2014. All Rights Reserved.

Thumb Motion Relative to the Upper Limb

Discrete CMC joint biomechanical studies eliminate the role of fingers, especially as targets and loading platforms for the thumb, and their positional change with different tasks. When either the elbow $[82,96]$ or wrist [40] is constrained, other joints compensate; by inference, joint contribution is determined for functional tasks. Electrogoniometric studies with a gimbal coordinate system provide estimates on elbow [76] and wrist [19, 84, 94] ROM for functional activities. The functional CMC joint range is harder to assess even with constraining experiments [43, $55,74]$, given the discrete but exacting motion of this joint and the functional restriction splints might impart [87].

Several of our experiments focus on analysis of thumb kinematics in relation to the upper limb. First, we 


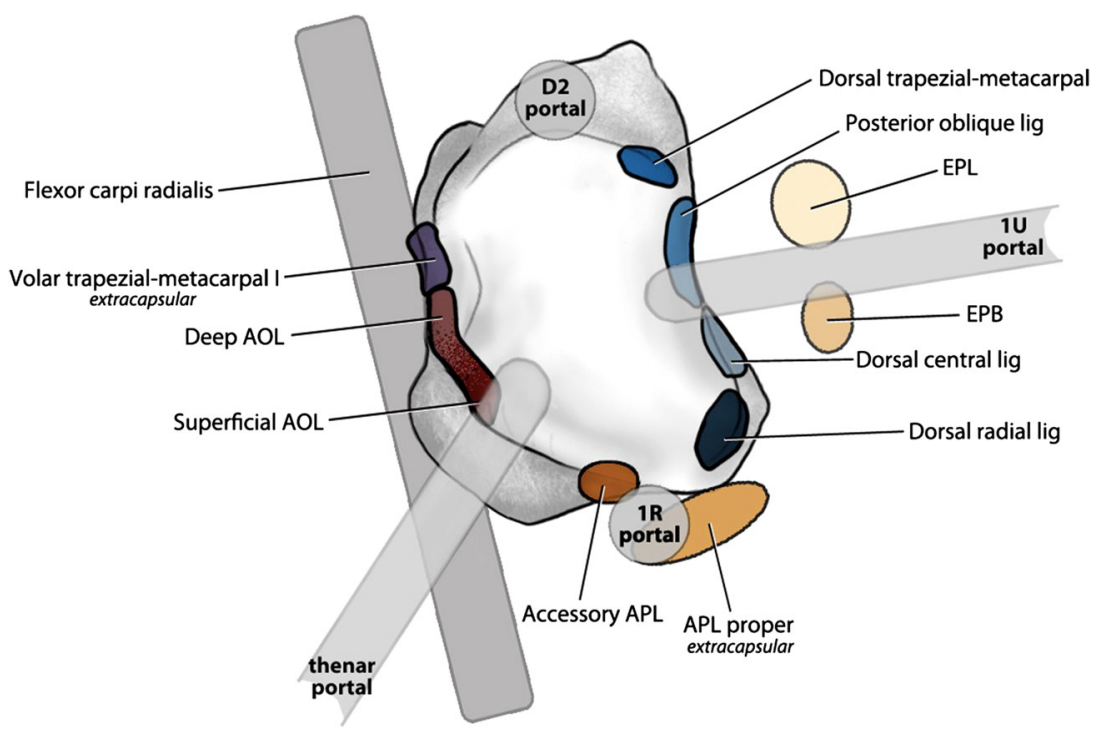

Fig. 7 The ligament and tendon location about the trapezium and metacarpal from arthroscopic specimens are illustrated as related to the common portals. Volar trapezial-metacarpal I is the same ligament as the ulnar collateral ligament (UCL) [108].
$\mathrm{AOL}=$ anterior oblique ligament; $\mathrm{APL}=$ abductor pollicis longus; $\mathrm{EPL}=$ extensor pollicis longus; $\mathrm{EPB}=$ extensor pollicis brevis. Published with kind permission of (C) S. Hegmann 2014. All Rights Reserved. simplified the coordinate system and image capture by modeling the phasic and relatively uniplanar orthograde nature of lower limb gait. We reported the upper limb kinematics of reach and grasp in 25 children with normal function and 12 children with spastic hemiplegia [22, 23]. The hemiplegic subjects demonstrated a contracted workspace. Compensatory trunk flexion, shoulder abduction, and forearm supination accompanied the contracted elbow and flexed wrist, and overall movements took longer, had more erratic trajectories, and lacked clear delineation of acceleration at midrange and deceleration at contact. An additional study of phasic golf swing [72] analyzed the interrelationship of the trunk, shoulder, upper limb, and wrist throughout the swing, with timing, wrist position, and peak torque reproducible among elite golfers. Amateur golfers had inconsistencies in timing, sequence, and reproducibility similar to the deviations seen with hemiplegic subjects. These studies provide the basis for examining the discrete, multiplanar tasks coordinating large and small joints of the upper limb.

To incorporate the CMC joint in upper limb analysis, we report a recent kinematic in vivo pilot study [67]. The functional tasks of grasp, jar opening, and key pinch, with and without loading were quantified in five asymptomatic women aged 25 to 35 years, using the same jigs from the CT kinematic study defining spatial registration and effort measurement. Using both close-range and traditional ceiling cameras, novel markers, and novel software application (UETrak, Motion Analysis Corp, Santa Rosa, CA, USA), we investigated simultaneous small joint and upper limb tracking (Fig. 15A-B) (Video 4; Supplemental material is available with the online version of $C O R R^{\circledR}$ ). The activities at rest and $80 \%$ effort were compared to those of a female subject with radiographic Eaton Stage IV [32] CMC OA. For each functional loaded task, the adducted metacarpal showed little movement; the MP hyperextended with compensatory abduction of the remaining thumb; and the wrist, elbow, and shoulder demonstrated compensatory movements compared to normal subjects. This included increased MP and interphalangeal flexion, deviation of the wrist, flexion of the elbow, and abduction of the shoulder to increase power generation. She took longer to complete the task and fatigued easily with accompanying progressive pain.

\section{Next Steps}

Prediction of presence and progression of arthritis, as well as improved means to treat it, may be addressed with computational modeling that incorporates kinetic and kinematic data. Traditional cadaveric biomechanical studies approximate the force on a joint via applied extrinsic and intrinsic tendon loads and employing mechanical models with simplified joint constructs [26, 49, 51]. From such models, we understand the progressive force increase from the tip to the CMC joint, with the force across the $\mathrm{CMC}$ joint in grasp exceeding that of lateral pinch 


\section{Trapezium Wear Patterns}

Double Saddle

Top Profile

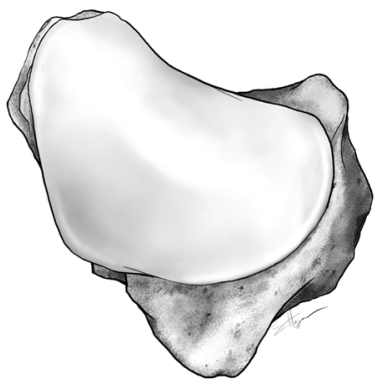

Side Profile

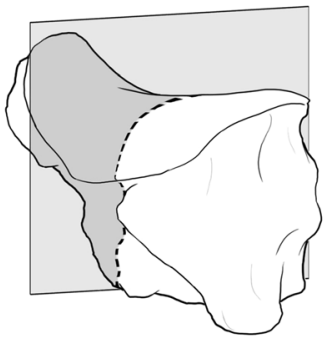

Dish
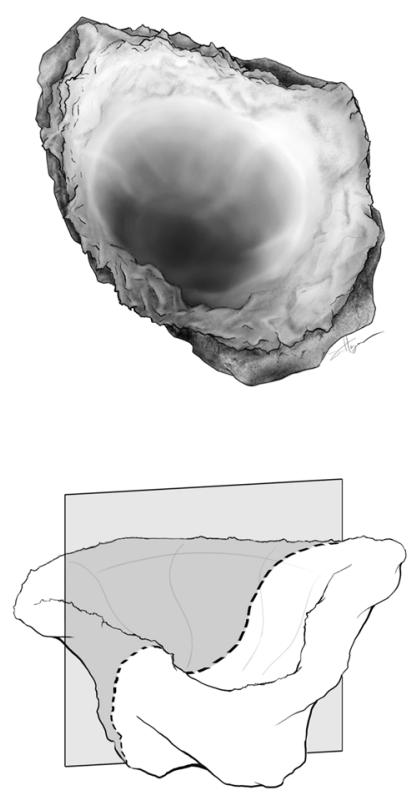

Cirque
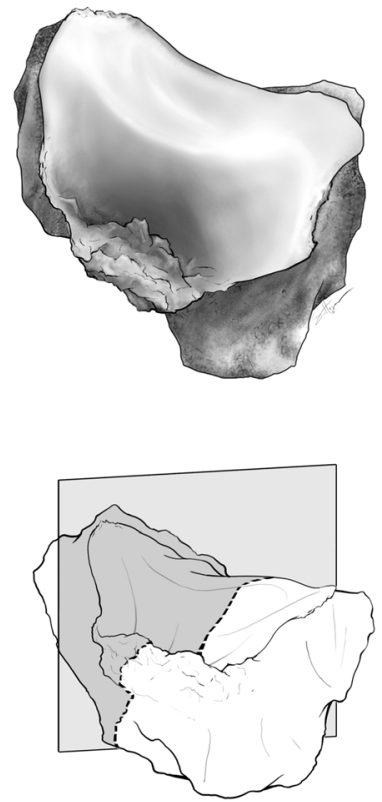

Schematic Representation

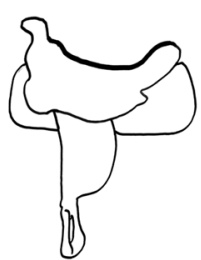

Fig. 8 The three patterns of wear from 36 explanted surgical specimens are illustrated: the retained convex-concave saddle; the concave dish with substantial rimming osteophytes; and the cirque [103]. The cirque pattern is named for the extra lip in a glacial mountaintop formation, often seen at a headwall on a ski slope. The

approximately 10 -fold (120 kg versus $12 \mathrm{~kg}$ ) [25]. New methods incorporate historical information and contributions from our work using CT, MR, and live kinematic data of divergent geometric bone patterns, joint contact, and movement to predict contact pressures between general 3-D surfaces or to predict functional movement and wear. We are pursuing these with collaborations at Brown University and University of Auckland, including the potential use of high-resolution registration with dual fluoroscopy $[73,104]$. The computational methods employ either statistical shape modeling $[5,41,66]$ or rigid body dynamics [41], integrating 3-D data to develop subject-specific models to predict changes over time, such as patterns of joint degeneration, response to fracture, or ligament disruption.
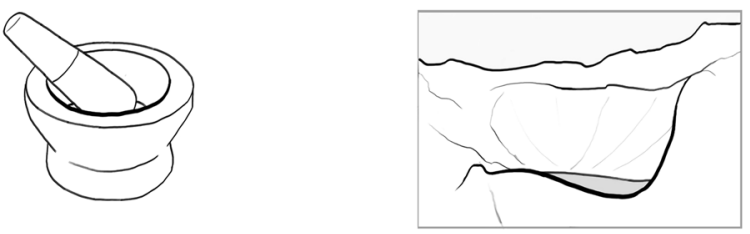

cirque varies in the width of the volar facet as either a small presence at the volar lip or expanding beyond the midline of the radial-ulnar axis. Published with kind permission of (c) S. Hegmann 2014. All Rights Reserved.

For motion analysis, the complexity of quantifying nonphasic functional activity in a sphere of motion with task measured in a discrete area poses many challenges. We strive for incorporating real-time analysis of complex motion that is readily reproduced and reproducible to quantify motion in congenital anomalies, neurologic and musculoskeletal injuries, and arthritis. Our arthritic subject in the kinematic study responded to visual coaching by reviewing the animation, and subsequent trials produced no compensation. We have pursued pilot studies to create biofeedback tools, including auditory feedback with sonification [28] for the golf swing, cerebral palsy, and the visual and performing arts [100]. Visual and auditory feedback suggests possible aids for rehabilitation or retraining to correct or restore mechanics associated with imbalance, disease, or injury. We have 

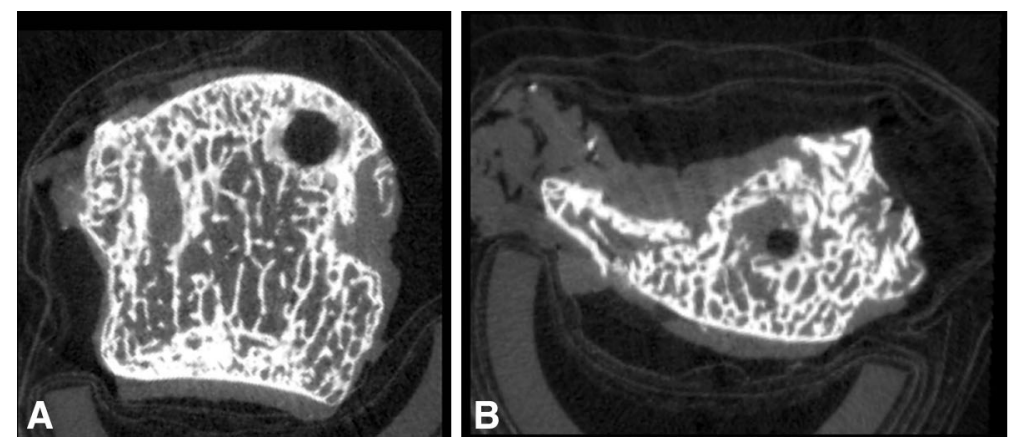

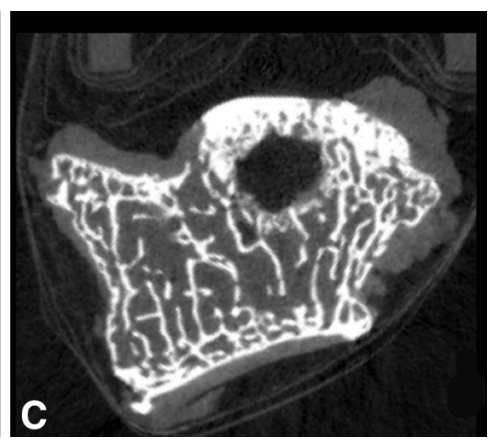

represents the core made from a tap used to remove the trapezium [103]. Published with kind permission of (C) AL Ladd, 2014. All Rights Reserved.

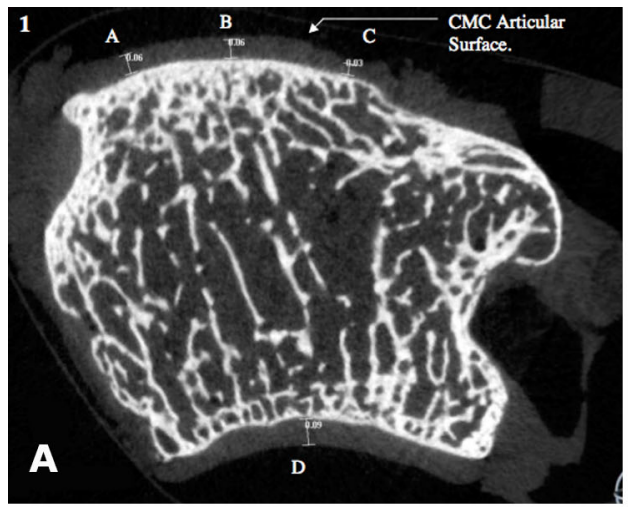

dorsal (left to right) plane demonstrate the convex configuration of (A) a saddle, (B) dish, and (C) cirque. The radiodense circle

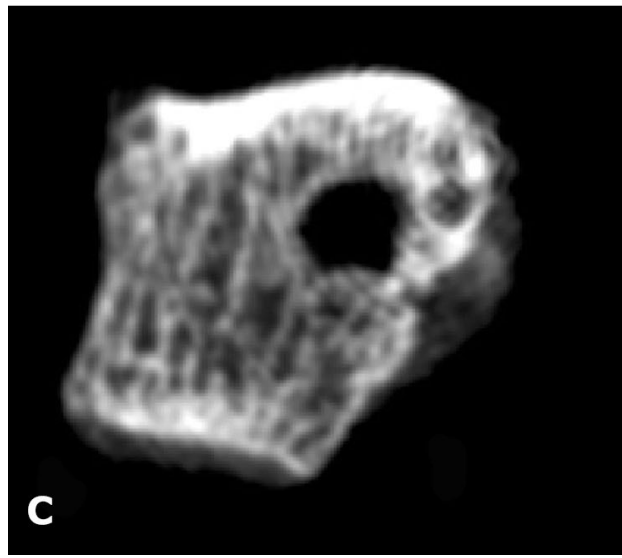

Fig. 10A-D Images of a trapezium are compared between (A) micro-CT to (B) histology and (C) portable flat panel CT scanner and (D) micro-CT. Toluidine blue represents preferential cartilage staining with histology. The cartilage thickness and the trabecular pattern are comparable between the different modalities. The trapezial

explored using accelerometers as joint sensors, EMG coupling with motion analysis, and a data glove to either supplement or replace the cumbersome motion analysis
2

B
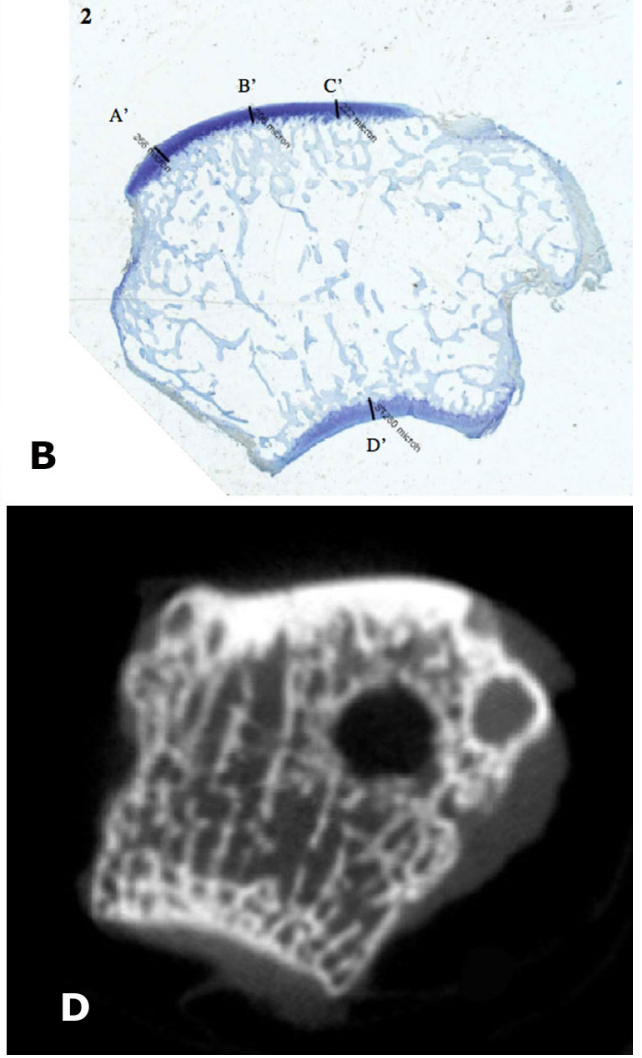

cartilage is quite thin, and it is difficult to ascertain its thickness even in micro-CT, but it compares favorably to histology, as does trabecular patterning. The flat panel scanner has the potential to image live subjects and thus image the articulating metacarpal and adjacent joints with microscopic detail not previously attained.

setup [100]. Technical considerations of size, ease of use, and quantifying fine coordinated motion required in a discrete area remain a challenge. 

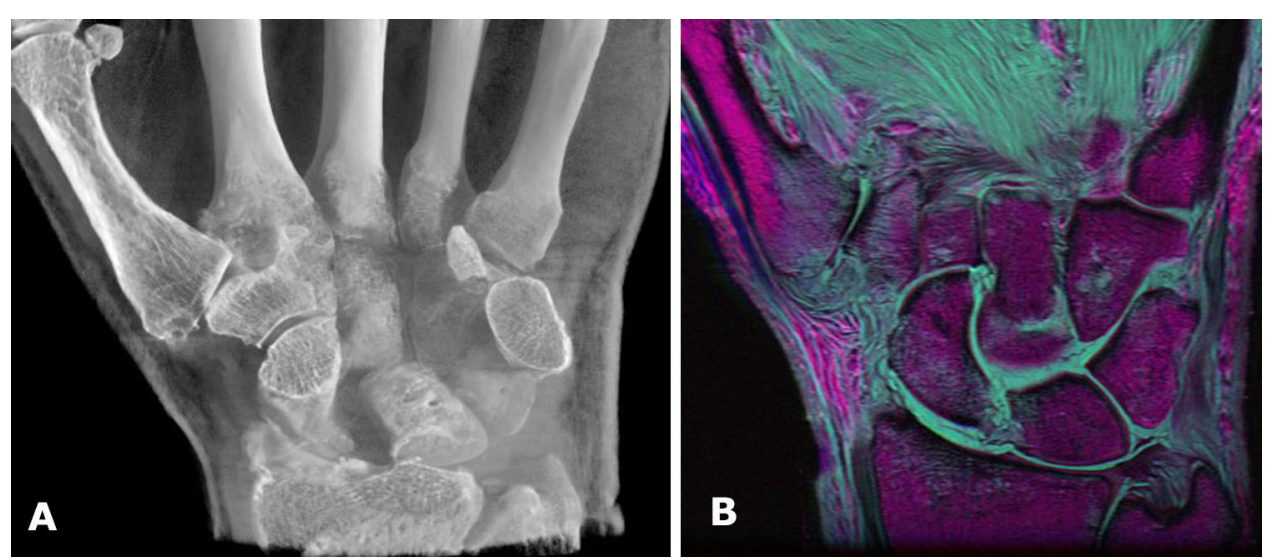

Fig. 11A-B (A) A CT scan is compared to (B) a false-color enhancement MRI in a cadaver with CMC OA, using the T1, T2, and proton density parameters to be reassigned as the red, green, blue channels as histograms (Beltrame). The fine trabeculae are seen in both images; the MRI demonstrates redundant dorsal-radial ligaments. Correlating scans on the MRI with the false-color assignment shows signal intensity differential on the ulnar portion of the metacarpal in the absence of obvious trabecular changes observed on CT. We hypothesize that this may represent a biochemical aberration, such as edema, abnormal crosslinking of collagen, or matrix pathology found in subchondral bone of OA.

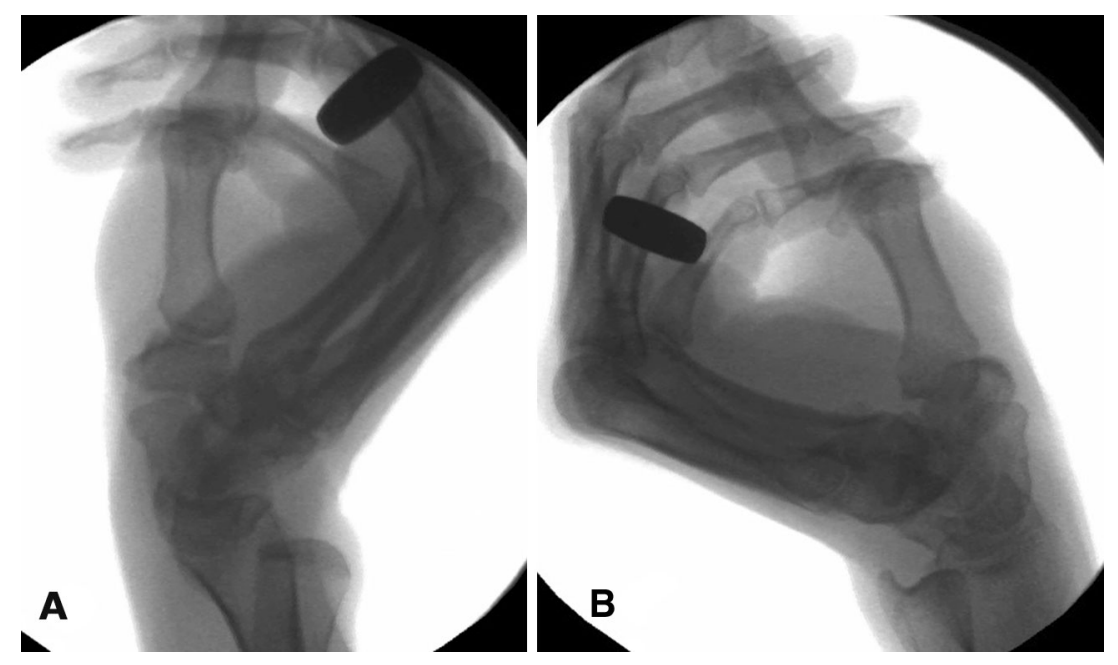

Fig. 12A-B (A) Lateral and (B) AP fluoroscopy images show a normal hand in grasp. The compression at the joint and the CMC motion is evident with load, but its precise position, translation, and rotation are not easily quantified given superimposition of structures and surfaces.

team is incorporating "nice to look at but not very practical" images of a decade ago into cross-platform, easyaccess technology. This permits recycling and repurposing content once acquired for proof of concept to accessible viewing and interactivity-from a 2000-year-old mummy [92], 200-year-old anatomic waxworks [99], and 75-yearold Bassett stereo anatomy photographs [98]; developing physical teaching models of fingers and tendons from CTgenerated data to recreate a cadaver demonstration from Dr. Robert Chase's 1959 video [100]; and reinvigorating first- and second-generation educational CD-ROMs, DVDs, and web modules [57, 59-61, 101]. The kinematic 
data from our CT micromotion study can be generated into an interactive movie today (Fig. 16A-D) (Video 5; Supplemental material is available with the online version of $\left.C O R R^{\circledR}\right)$. We have created templates of interactive educational modules from these rich data resources, most

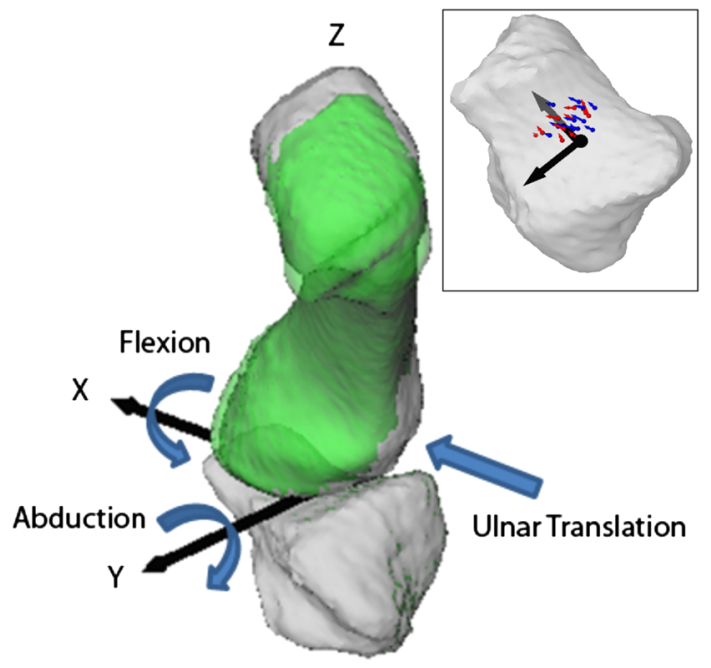

Fig. 13 With markerless bone registration kinematic analysis of loaded object grasp, the thumb metacarpal undergoes ulnar translation, flexion, and abduction relative to the trapezium. With loaded key pinch (not shown), the thumb metacarpal undergoes volar translation, internal rotation, and flexion relative to the trapezium. Published with kind permission of (C) Brown University Orthopaedics Engineering Laboratory, 2014. All Rights Reserved. recently targeting "smart" handheld devices across platforms.

\section{Discussion}

Recent technological developments have permitted improved acquisition and interpretation of the morphology, load, and motion of the thumb, especially in live subjects, which provide clues to the complexity of the thumb as an organ system. The ultimate goal is to decode the action of the musculoskeletal system in both the normal and pathologic states across a spectrum of human development, ethnic diversity, and sex differences. Our investigations in this article emphasizing thumb CMC OA and its pathophysiology have been possible with recent advances in computational power, imaging capabilities, and software enhancements in the realm of "disruptive innovation" [24] that create new opportunities for acquisition and dissemination of information.

Additional studies in the realm of multimodal technology that define patterns of normal use and wear will provide data to better characterize CMC OA and opportunities for tailored treatment, including prevention, delay of progression, and joint arthroplasty. Such innovation allows us to expand on current methodologies and refurbish others so that deciphering the puzzle of the thumb-in all its beauty and weakness-is well within our grasp.

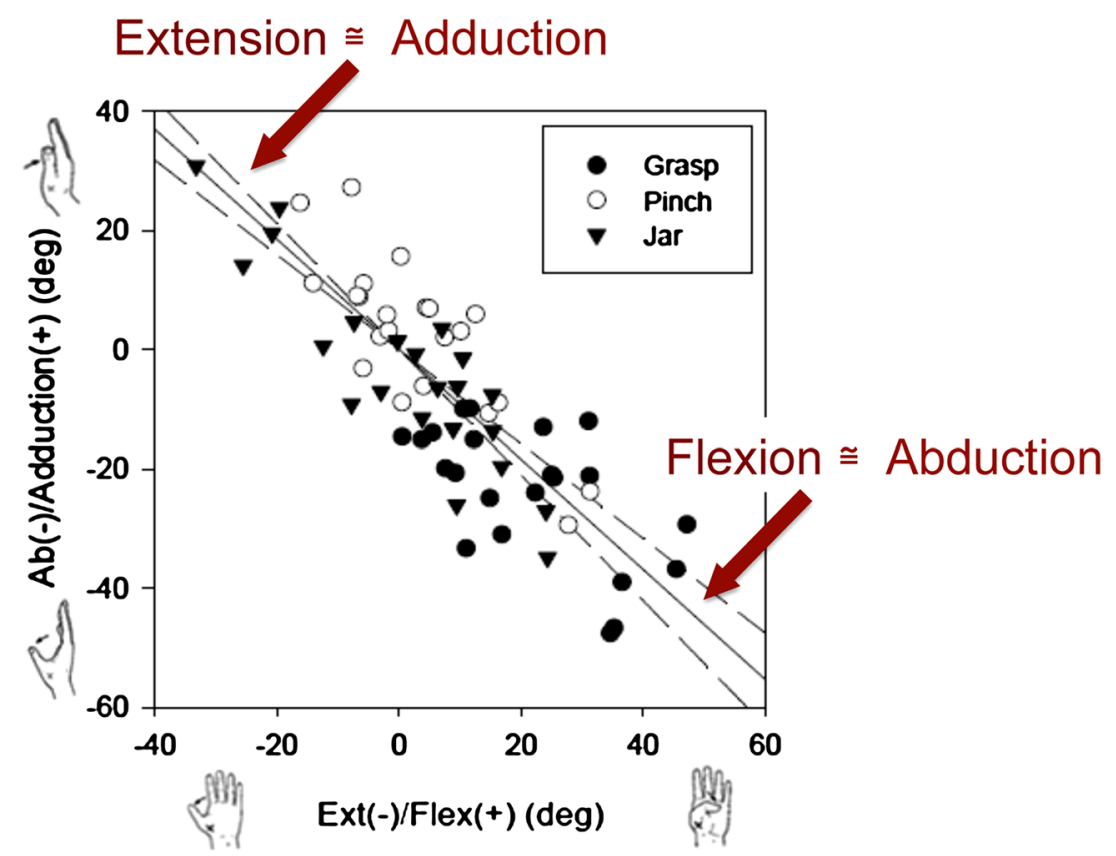

Fig. 14 A specific CMC functional coupling occurs in multiple tasks. Extension of the thumb metacarpal relative to the trapezium couples with adduction and flexion couples with abduction. Published with kind permission of (C) Brown University Orthopaedics Engineering Laboratory, 2014. All Rights Reserved. 


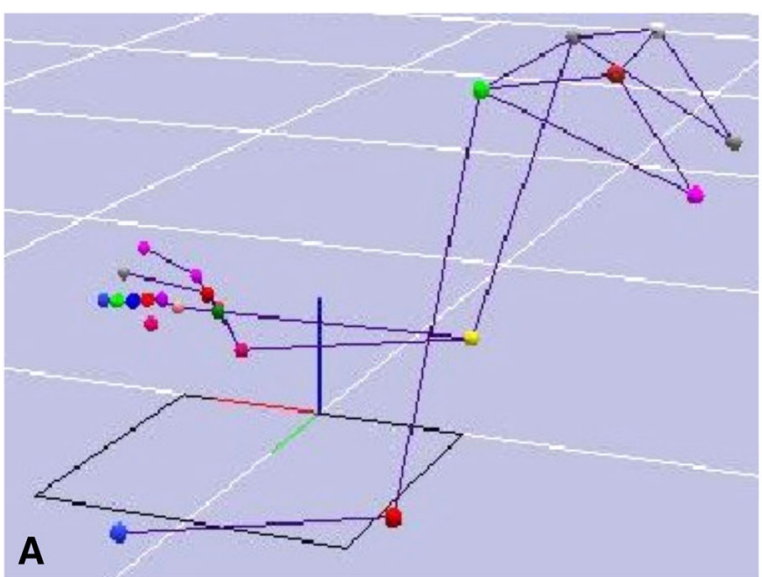

Fig. 15A-B Motion capture of jar opening is shown. (A) The reflective markers are placed on the thumb ray, wrist, index and small fingers, forearm, elbow, and shoulder. (B) The CMC and thumb ray

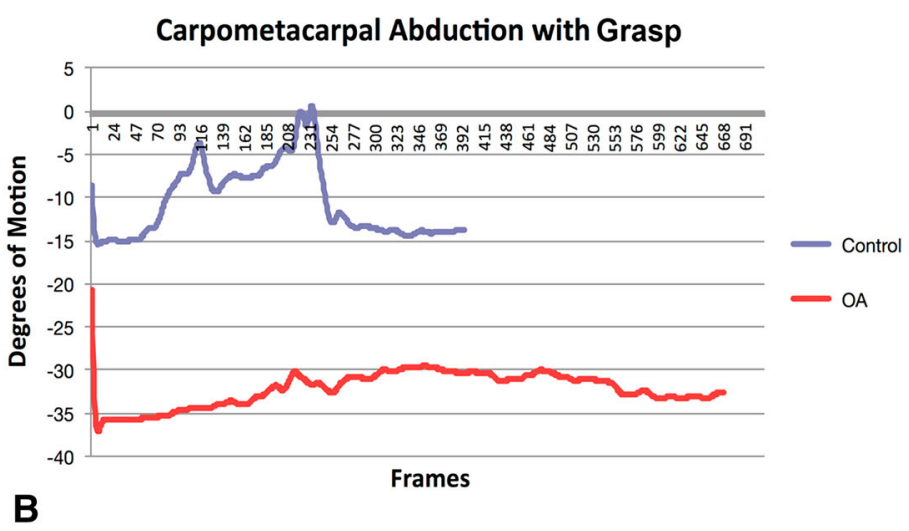

demonstrate minimal abduction in a subject with OA compared to an asymptomatic control.
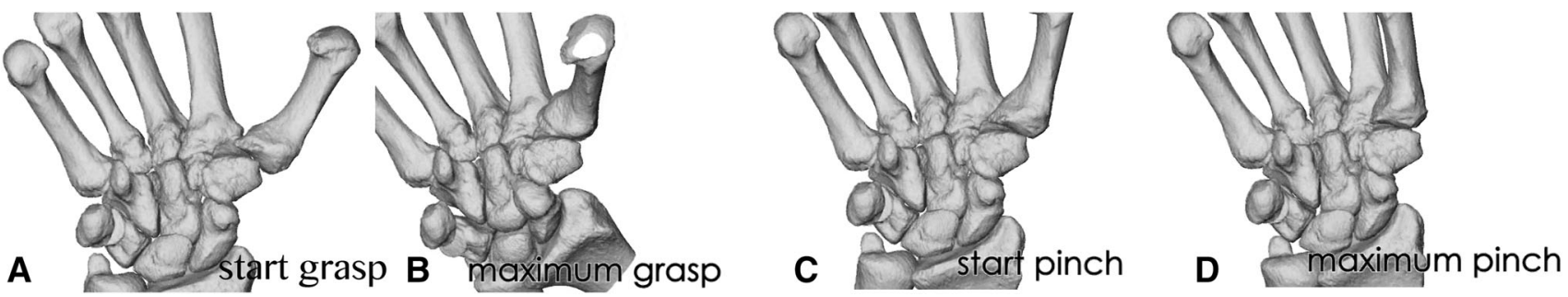

Fig. 16A-D Still shots of the hand demonstrate $(\mathbf{A})$ grasp, $(\mathbf{B})$ jar opening, and $(\mathbf{C}, \mathbf{D})$ lateral pinch in an asymptomatic subject as recreated from the surface-rendered CT kinematic models. The use of slider bars and pause button permits some level of interactivity.

Acknowledgments The breadth of collaborative colleagues includes medical students, graduate students, residents; colleagues at the Stanford University Chase Hand Center, Department of Orthopaedic Surgery, Division of Anatomy, Department of Radiology, Department of Bioengineering; Stanford University Anatomy of Movement class; Brown University; and the Karolinska Institutet. Dr. Robert Chase's lifelong pursuit of teaching human anatomy, with special emphasis on the thumb, represents the primary inspiration for this work.

\section{References}

1. Alba DM, Moya-Sola S, Kohler M. Morphological affinities of the Australopithecus afarensis hand on the basis of manual proportions and relative thumb length. J Hum Evol. 2003;44:225-254.

2. Almecija S, Moya-Sola S, Alba DM. Early origin for humanlike precision grasping: a comparative study of pollical distal phalanges in fossil hominins. PLoS One. 2010;5:e11727.

3. Ateshian GA, Ark JW, Rosenwasser MP, Pawluk RJ, Soslowsky LJ, Mow VC. Contact areas in the thumb carpometacarpal joint. J Orthop Res. 1995;13:450-458.

4. Ateshian GA, Rosenwasser MP, Mow VC. Curvature characteristics and congruence of the thumb carpometacarpal joint: differences between female and male joints. J Biomech. 1992;25:591-607.
5. Baldwin MA, Langenderfer JE, Rullkoetter PJ, Laz PJ. Development of subject-specific and statistical shape models of the knee using an efficient segmentation and mesh-morphing approach: computer methods and programs in biomedicine. Comput Methods Programs Biomed. 2010;97:232-240.

6. Beck JD, Canfield BL, Haddock SM, Chen TJ, Keaveny TM. Three-dimensional imaging of trabecular bone using the computer numerically controlled milling technique. Bone. 1997;21:281-287.

7. Bell C. An idea of a new anatomy of the brain; submitted for the observations of his friends. 1868. Available at: http://www.ncbi. nlm.nih.gov/pmc/articles/PMC1318665/pdf/janatphys00211-0151. pdf. Accessed 11 June 2014.

8. Bell C. On the nervous circle which connects the voluntary muscles with the brain. Philos Trans $R$ Soc Lond. 1826;116: $1-36$.

9. Bell C. The Hand_Its Mechanism and Vital Endowments as Evincing Design. 2nd ed. London, UK: William Pickering; 1833.

10. Beltrame F, Fato M, Raposio E, Sobel I. Recent results in color compositing of three-parameter magnetic resonance scans as a preoperative aid to the management of upper limb sarcomas. MAGMA. 1997;5:289-298.

11. Berger AJ, Momeni A, Ladd AL. Intra- and interobserver reliability of the Eaton classification for trapeziometacarpal arthritis: a systematic review. Clin Orthop Relat Res. 2014; 472:1155-1159. 
12. Bettinger PC, Linscheid RL, Berger RA, Cooney WP 3rd, An $\mathrm{KN}$. An anatomic study of the stabilizing ligaments of the trapezium and trapeziometacarpal joint. J Hand Surg Am. 1999;24:786-798.

13. Bettinger PC, Linscheid RL, Cooney WP 3rd, An KN. Trapezial tilt: a radiographic correlation with advanced trapeziometacarpal joint arthritis. J Hand Surg Am. 2001;26:692-697.

14. Blauth W, Schneider-Sickert F. Numerical variations. In: Blauth W, Schneider-Sickert F, eds. Congenital Deformities of the Hand: An Atlas on Their Surgical Treatment. Berlin, Germany: Springer-Verlag; 1981:119-123.

15. Bojsen-Møller F. Calcaneocuboid joint and stability of the longitudinal arch of the foot at high and low gear push off. $J$ Anat. 1979;129(pt 1):165-176.

16. Bojsen-Møller F. Osteoligamentous guidance of the movements of the human thumb. Am J Anat. 1976;147:71-80.

17. Boyd SK, Muller R, Leonard T, Herzog W. Long-term periarticular bone adaptation in a feline knee injury model for posttraumatic experimental osteoarthritis. Osteoarthritis Cartilage. 2005;13:235-242.

18. Boyd SK, Muller R, Zernicke RF. Mechanical and architectural bone adaptation in early stage experimental osteoarthritis. $J$ Bone Miner Res. 2002;17:687-694.

19. Brumfield RH, Champoux JA. A biomechanical study of normal functional wrist motion. Clin Orthop Relat Res. 1984;187: $23-25$.

20. Buck-Gramcko D. Congenital malformations of the hand and forearm. Chir Main. 2002;21:70-101.

21. Bunnell S. Surgery of the Hand. 2nd ed. Philadelphia, PA: Lippincott; 1948.

22. Butler EE, Ladd AL, Lamont LE, Rose J. Temporal-spatial parameters of the upper limb during a Reach \& Grasp Cycle for children. Gait Posture. 2010;32:301-306.

23. Butler EE, Ladd AL, Louie SA, Lamont LE, Wong W, Rose J. Three-dimensional kinematics of the upper limb during a Reach and Grasp Cycle for children. Gait Posture. 2010;32:72-77.

24. Christensen CM. The Innovator's Solution: Creating and Sustaining Successful Growth. Boston, MA: Harvard Business Press; 2003.

25. Cooney WP 3rd, Chao EY. Biomechanical analysis of static forces in the thumb during hand function. J Bone Joint Surg Am. 1977;59:27-36.

26. Cooney WP 3rd, Lucca MJ, Chao EY, Linscheid RL. The kinesiology of the thumb trapeziometacarpal joint. J Bone Joint Surg Am. 1981;63:1371-1381.

27. Crisco JJ, Coburn JC, Moore DC, Akelman E, Weiss AP, Wolfe SW. In vivo radiocarpal kinematics and the dart thrower's motion. J Bone Joint Surg Am. 2005;87:2729-2740.

28. Delogu F, Palmiero M, Federici S, Plaisant C, Zhao H, Belardinelli O. Non-visual exploration of geographic maps: does sonification help? Disabil Rehabil Assist Technol. 2010;5: 164-174.

29. Ding M, Odgaard A, Hvid I. Changes in the three-dimensional microstructure of human tibial cancellous bone in early osteoarthritis. J Bone Joint Surg Br. 2003;85:906-912.

30. Du Bois-Reymond R. [The saddle joint. Archives of Anatomy and Physiology (Physiological Division)] [in German]. $1895 ; 433-462$.

31. Dye SF. An evolutionary perspective of the knee. J Bone Joint Surg Am. 1987;69:976-983.

32. Eaton RG, Glickel SZ. Trapeziometacarpal osteoarthritis: Staging as a rationale for treatment. Hand Clin. 1987;3: 455-471.

33. Eaton RG, Lane LB, Littler JW, Keyser JJ. Ligament reconstruction for the painful thumb carpometacarpal joint: a longterm assessment. J Hand Surg Am. 1984;9:692-699.
34. Eaton RG, Littler JW. A study of the basal joint of the thumb: treatment of its disabilities by fusion. $J$ Bone Joint Surg Am. 1969;51:661-668.

35. Eaton RG, Littler JW. Ligament reconstruction for the painful thumb carpometacarpal joint. J Bone Joint Surg Am. 1973;55: $1655-1666$.

36. Edmunds JO. Traumatic dislocations and instability of the trapeziometacarpal joint of the thumb. Hand Clin. 2006;22: 365-392.

37. Fick A. [The joints with saddle shaped areas. Journal of Rational Medicine] [in German]. 1854;314-321.

38. Fick R. [Special joint and muscle mechanics. In: von Bardeleben K, ed. Handbook of Human Anatomy] [in German]. Jena, Germany: Gustav Fischer; 1911:401-405.

39. Flower WH. An Introduction to the Osteology of the Mammalia. 2nd ed. London, UK: Macmillan and Co; 1876.

40. Franko OI, Lal S, Pauyo T, Alexander M, Zurakowski D, Day C. Validation of an objective device for assessing circumductive wrist motion. J Hand Surg Am. 2008;33:1293-1300.

41. Fregly BJ, Bei Y, Sylvester ME. Experimental evaluation of an elastic foundation model to predict contact pressures in knee replacements. J Biomech. 2003;36:1659-1668.

42. Garcia-Elias M, Orsolini C. Relationship between thumb laxity and trapezium kinematics. Chir Main. 2011;30:224-227.

43. Gehrmann SV, Tang J, Li ZM, Goitz RJ, Windolf J, Kaufmann RA. Motion deficit of the thumb in CMC joint arthritis. $J$ Hand Surg Am. 2010;35:1449-1453.

44. Gray H, Lewis WH (ed). Anatomy of the Human Body. 20th ed. Philadelphia, PA: Lea \& Febiger; 1918.

45. Hagert E, Lee J, Ladd AL. Innervation patterns of the thumb trapeziometacarpal joint ligaments. $J$ Hand Surg Am. 2012;37:706-714.

46. Haines RW. The mechanism of rotation at the first carpometacarpal joint. J Anat. 1944;78:44-46.

47. Halilaj E, Rainbow MJ, Got C, Schwartz JB, Moore DC, Weiss AP, Ladd AL, Crisco JJ. In vivo kinematics of the thumb carpometacarpal joint during three isometric functional tasks. Clin Orthop Relat Res. 2014;472:1114-1122.

48. Hildebrand T, Laib A, Muller R, Dequeker J, Ruegsegger P. Direct three-dimensional morphometric analysis of human cancellous bone: microstructural data from spine, femur, iliac crest, and calcaneus. J Bone Miner Res. 1999;14:1167-1174.

49. Imaeda T, An KN, Cooney WP 3rd. Functional anatomy and biomechanics of the thumb. Hand Clin. 1992;8:9-15.

50. Imaeda T, An KN, Cooney WP 3rd, Linscheid R. Anatomy of trapeziometacarpal ligaments. J Hand Surg Am. 1993;18: 226-231.

51. Imaeda T, Niebur G, Cooney WP 3rd, Linscheid RL, An KN. Kinematics of the normal trapeziometacarpal joint. J Orthop Res. 1994;12:197-204.

52. Kivell TL, Kibii JM, Churchill SE, Schmid P, Berger LR. Australopithecus sediba hand demonstrates mosaic evolution of locomotor and manipulative abilities. Science. 2011;333: $1411-1417$.

53. Koff MF, Ugwonali OF, Strauch RJ, Rosenwasser MP, Ateshian GA, Mow VC. Sequential wear patterns of the articular cartilage of the thumb carpometacarpal joint in osteoarthritis. $J$ Hand Surg Am. 2003;28:597-604.

54. Kovler M, Lundon K, McKee N, Agur A. The human first carpometacarpal joint: osteoarthritic degeneration and 3dimensional modeling. J Hand Ther. 2004;17:393-400.

55. Kuczynski K. Carpometacarpal joint of the human thumb. $J$ Anat. 1974;118(pt 1):119-126.

56. Ladd AL. Thoughts on the accomplishment and possibility of the human hand: Sterling Bunnell traveling fellow report 2000-2001. J Am Soc Surg Hand. 2003;3:117-128. 
57. Ladd AL. Thumb CMC Arthritis: A Patient Resource. Stanford, CA: Stanford Clinical Anatomy;2013:1-49.

58. Ladd AL. Upper limb evolution and development: skeletons in the closet—congenital anomalies and evolution's template. $J$ Bone Joint Surg Am. 2009;91(suppl 4):19-25.

59. Ladd AL, Jones HH, Otañez O. Osteomyelitis. February 2003. Available at: http://osteomyelitis.stanford.edu. Accessed 11 June 2014.

60. Ladd AL, Jones HH, Otañez O. Osteosarcoma. November 2003. Available at: http://osteosarcoma.stanford.edu. Accessed 11 June 2014.

61. Ladd AL, Jones HH, Otañez O. Paget's disease of bone. November 2002. Available at: http://paget.stanford.edu. Accessed 11 June 2014.

62. Ladd AL, Lee J, Hagert E. Macroscopic and microscopic analysis of the thumb carpometacarpal ligaments: A cadaveric study of ligament anatomy and histology. J Bone Joint Surg Am. 2012;94:1468-1477.

63. Ladd AL, Weiss AP, Crisco JJ, Hagert E, Wolf JM, Glickel SZ, Yao J. The thumb carpometacarpal joint: anatomy, hormones, and biomechanics. Instr Course Lect. 2013;62:165-179.

64. Lee AT, Williams AA, Lee J, Cheng R, Lindsey DP, Ladd AL. Trapezium trabecular morphology in carpometacarpal arthritis. $J$ Hand Surg Am. 2013;38:309-315.

65. Lee J, Ladd A, Hagert E. Immunofluorescent triple-staining technique to identify sensory nerve endings in human thumb ligaments. Cells Tissues Organs. 2012;195:456-464.

66. Li L, Patil S, Steklov N, Bae W, Temple-Wong M, D'Lima DD, Sah RL, Fregly BJ. Computational wear simulation of patellofemoral articular cartilage during in vitro testing. $J$ Biomech. 2011;44:1507-1513.

67. Luker KR, Aguinaldo A, Kenney D, Cahill-Rowley K, Ladd AL. Functional task kinematics of the thumb carpometacarpal joint. Clin Orthop Relat Res. 2014;472:1123-1129.

68. Manske PR, McCarroll HR Jr, James MA. Type III-A hypoplastic thumb. J Hand Surg Am. 1995;20:246-253.

69. Marzke MW. Evolutionary development of the human thumb. Hand Clin. 1992;8:1-8.

70. Marzke MW, Tocheri MW, Marzke RF, Femiani JD. Threedimensional quantitative comparative analysis of trapezialmetacarpal joint surface curvatures in human populations. $J$ Hand Surg Am. 2012;37:72-76.

71. Marzke MW, Tocheri MW, Steinberg B, Femiani JD, Reece SP, Linscheid RL, Orr CM, Marzke RF. Comparative 3D quantitative analyses of trapeziometacarpal joint surface curvatures among living catarrhines and fossil hominins. Am J Phys Anthropol. 2010;141:38-51.

72. Meister D, Ladd AL, Butler EE, Rogers A, Ray C, Rose J. Rotational biomechanics of the elite golf swing: benchmarks for amateurs. J Appl Biomech. 2011;27:242-251.

73. Miranda DL, Rainbow MJ, Leventhal EL, Crisco JJ, Fleming BC. Automatic determination of anatomical coordinate systems for three-dimensional bone models of the isolated human knee. $J$ Biomech. 2010;43:1623-1626.

74. Miura T, Ohe T, Masuko T. Comparative in vivo kinematic analysis of normal and osteoarthritic trapeziometacarpal joints. $J$ Hand Surg Am. 2004;29:252-257.

75. Mobargha N, Ludwig C, Ladd AL, Hagert E. Ultrastructure and innervation of thumb carpometacarpal ligaments in surgical patients with osteoarthritis. Clin Orthop Relat Res. 2014;472:1146-1154

76. Morrey BF, Askew LJ, Chao EY. A biomechanical study of normal functional elbow motion. J Bone Joint Surg Am. 1981;63:872-877.

77. Napier JR. The form and function of the carpo-metacarpal joint of the thumb. J Anat. 1955;89:362-369.
78. Neumann DA, Bielefeld T. The carpometacarpal joint of the thumb: stability, deformity, and therapeutic intervention. $J$ Orthop Sports Phys Ther. 2003;33:386-399.

79. North ER, Rutledge WM. The trapezium-thumb metacarpal joint: the relationship of joint shape and degenerative joint disease. Hand. 1983;15:201-206.

80. Nufer P, Goldhahn J, Kohler T, Kuhn V, Muller R, Herren DB. Microstructural adaptation in trapezial bone due to subluxation of the thumb. J Orthop Res. 2008;26:208-216.

81. O'Brien VH, Giveans MR. Effects of a dynamic stability approach in conservative intervention of the carpometacarpal joint of the thumb: A retrospective study. $J$ Hand Ther. 2013;26:44-51.

82. O'Neill OR, Morrey BF, Tanaka S, An KN. Compensatory motion in the upper extremity after elbow arthrodesis. Clin Orthop Relat Res. 1992;281:89-96.

83. Outerbridge RE. The etiology of chondromalacia patellae. $J$ Bone Joint Surg Br. 1961;43:752-757.

84. Palmer AK, Werner FW, Murphy D, Glisson R. Functional wrist motion: a biomechanical study. $J$ Hand Surg Am. 1985;10:39-46.

85. Pellegrini VD Jr. Osteoarthritis of the trapeziometacarpal joint: the pathophysiology of articular cartilage degeneration. I. Anatomy and pathology of the aging joint. J Hand Surg Am. 1991;16:967-974.

86. Pellegrini VD Jr. Osteoarthritis of the trapeziometacarpal joint: the pathophysiology of articular cartilage degeneration. II. Articular wear patterns in the osteoarthritic joint. J Hand Surg Am. 1991;16:975-882.

87. Pellegrini VD Jr. The ABJS 2005 Nicolas Andry Award. Osteoarthritis and injury at the base of the human thumb: survival of the fittest? Clin Orthop Relat Res. 2005;438:266-276.

88. Pellegrini VD Jr, Olcott CW, Hollenberg G. Contact patterns in the trapeziometacarpal joint: the role of the palmar beak ligament. J Hand Surg Am. 1993;18:238-244.

89. Pellegrini VD Jr, Smith RL, Ku CW. Pathobiology of articular cartilage in trapeziometacarpal osteoarthritis. I. Regional biochemical analysis. J Hand Surg Am. 1994;19:70-78.

90. Poole JU, Pellegrini VD Jr. Arthritis of the thumb basal joint complex. J Hand Ther. 2000;13:91-107.

91. Robert P. [Bulletins and Memoirs of the Society of Medical Radiology of France] [in French]. 1936;24:687-690.

92. Rogers C. Tales from the Crypt: Stanford orthopaedists, others unravel mystery of 2,000-year-old child mummy. Available at: http://www2.aaos.org/bulletin/feb06/feature5.asp. Accessed 11 June 2014.

93. Rothschild BM, Rühli FJ. Comparison of arthritis characteristics in lowland Gorilla gorilla and mountain Gorilla beringei. Am J Primatol. 2005;66:205-218.

94. Ryu JY, Cooney WP 3rd, Askew LJ, An KN, Chao EY. Functional ranges of motion of the wrist joint. J Hand Surg Am. 1991;16:409-419.

95. Segura MJ, Gandolfo CN, Sica RE. Percutaneous cervical stimulation: effects on intraspinal structures. Electroencephalogr Clin Neurophysiol. 1991;81:299-303.

96. Snider WJ, DeWitt HJ. Functional study for optimum position for elbow arthrodesis of ankylosis. J Bone Joint Surg Am. 1973;55:1305.

97. Spitzer A, Claus D. The influence of the shape of mechanical stimuli on muscle stretch reflexes and SEP. Electroencephalogr Clin Neurophysiol. 1992;85:331-336.

98. Stanford Medicine. Lane medical library. Available at: http:// lane.stanford.edu/biomed-resources/bassett/raw/index.html. Accessed 11 June 2014.

99. Stanford University School of Medicine. Italian anatomical waxworks-timeless preservation with stereophotography. 
Available at: http://handsurgery.stanford.edu/projects/waxworks/. Accessed 11 June 2014.

100. Stanford University School of Medicine. The anatomy of movement. Available at: http://move.stanford.edu/09/index. html. Accessed 11 June 2014.

101. Steinmann SP, Ring DC, Ladd AL. Fractured Wrist Virtual Fellowship Interactive Multimedia Program [DVD-ROM]. Rosemont, IL: American Academy of Orthopaedic Surgeons; 2008.

102. Tocheri MW, Orr CM, Jacofsky MC, Marzke MW. The evolutionary history of the hominin hand since the last common ancestor of Pan and Homo. J Anat. 2008;212:544-562.

103. Van Nortwick S, Berger A, Cheng R, Lee J, Ladd AL. Trapezial topography in thumb carpometacarpal arthritis. J Wrist Surg. 2013;2:263-270.

104. Varadarajan KM, Freiberg AA, Gill TJ, Rubash HE, Li G. Relationship between three-dimensional geometry of the trochlear groove and in vivo patellar tracking during weightbearing knee flexion. J Biomech Eng. 2010;132:061008.

105. Wilson FR. The Hand: How Its Use Shapes the Brain, Language, and Human Culture. New York, NY: Pantheon Books; 1998.

106. Wolff J. [The Law of Transformation of Bone] [in German]. Berlin, Germany: Hirschwald; 1892.

107. Xu L, Strauch RJ, Ateshian GA, Pawluk RJ, Mow VC, Rosenwasser MP. Topography of the osteoarthritic thumb carpometacarpal joint and its variations with regard to gender, age, site, and osteoarthritic stage. J Hand Surg Am. 1998;23:454-464.

108. Zancolli EA, Ziadenberg C, Zancolli E Jr. Biomechanics of the trapeziometacarpal joint. Clin Orthop Relat Res. 1987;220:14-26.

109. Zhang AY, Van Nortwick S, Hagert E, Ladd A. Thumb CMC ligaments inside and out: a comparative study of arthroscopy and gross anatomy. J Wrist Surg. 2013;2:55-62. 